\title{
A CRUCIAL ROLE OF URIDINE/CYTIDINE KINASE 2 IN ANTITUMOR ACTIVITY OF 3'-ETHYNYL NUCLEOSIDES
}

\author{
Y. Endo, T. Obata, D. Murata, M. Ito, M. Kadohira and T. Sasaki
}

The antitumor 3'-ethynyl nucleosides, 1-(3-C-ethynyl- $\beta$-D-ribopentofuranosyl)cytosine (ECyd) and 1-(3-C-ethynyl- $\beta$-D-ribopentofuranosyl)uracil (EUrd), are potent inhibitors of RNA polymerases and show excellent antitumor activity against various human solid tumors in xenograft models. ECyd is being investigated in phase I clinical trials as a novel anticancer drug possessing a unique antitumor action. ECyd and EUrd require the activity of uridine/cytidine kinase (UCK) to produce the corresponding active metabolite (ECTP and EUTP). The UCK family consists of two members, UCK1 and UCK2, and both UCKs are expressed in many tumor cells. It was unclear, however, whether UCK1 or UCK2 is responsible for the phosphorylation of the 3'-ethynyl nucleosides. We therefore established cell lines that are highly resistant to the 3'-ethynyl nucleosides from human fibrosarcoma HT-1080 and gastric carcinoma NUGC-3. All the resistant cell lines showed a high cross-resistance to ECyd and EUrd. As a result of cDNA sequence analysis, we found that UCK2 mRNA expressed in EUrd-resistant HT-1080 cells has a 98-base pair deletion of exon 5, whereas EUrd-resistant NUGC-3 cells were harboring the point mutation at nucleotide position 484 ( $\mathrm{C}$ to $\mathrm{T}$ ) within exon 4 of UCK2 mRNA. This mutation was confirmed by genome sequence analysis of the UCK2 gene. In ECyd-resistant NUGC-3 cells, the substitution of G to A at $391 \mathrm{nt}$ of cording region of UCK2 cDNA (Val to Met at codon 131) was found. Moreover, the expression of UCK2 protein and the enzymatic activity were decreased in these resistant cells. In contrast, no mutation in the mRNA or differences in protein expression levels of UCK1 were shown in all the resistant cells. These results suggest that UCK2 is responsible for the phosphorylation and activation of the antitumor 3 '-ethynyl nucleosides. Although UCK2 is expressed in many types of tissues and tumor cells, the expression level of UCK2 appears to be very diverse in cells. UCK2 may be a useful marker to predict chemosensitivity to antitumor uridine and cytidine analogs including EUrd and ECyd. In addition, UCK2 may be a novel molecular target for biochemical modulation to potentiate antitumor effects of such nucleoside analogs.

\section{References}

1. Murata D, Endo Y, Obata T, Sakamoto K, Syouji Y, Kadohira M, Matsuda A, Sasaki T. (2004) A crucial role of uridine/cytidine kinase 2 in antitumor activity of 3'-ethynyl nucleosides. Drug Metab Dispos. 32(10):1178-82.

2. Tabata S, Tanaka M, Endo Y, Obata T, Matsuda A, Sasaki T. (1997) Anti-tumor mechanisms of 3'-ethynyluridine and 3'-ethynylcytidine as RNA synthesis inhibitors: development and characterization of 3'-ethynyluridine-resistant cells. Cancer Lett. 116(2):225-31. 